\title{
Remote monitoring system for real time detection and classification of transmission line faults in a power grid using PMU measurements
}

\author{
Pathirikkat Gopakumar ${ }^{1}$, Balimidi Mallikajuna², Maddikara Jaya Bharata Reddy ${ }^{2 *}$ and Dusmanta Kumar Mohanta ${ }^{3}$
}

\begin{abstract}
Remote monitoring of transmission lines of a power system is significant for improved reliability and stability during fault conditions and protection system breakdowns. This paper proposes a smart backup monitoring system for detecting and classifying the type of transmission line fault occurred in a power grid. In contradiction to conventional methods, transmission line fault occurred at any locality within power grid can be identified and classified using measurements from phasor measurement unit (PMU) at one of the generator buses. This minimal requirement makes the proposed methodology ideal for providing backup protection. Spectral analysis of equivalent power factor angle (EPFA) variation has been adopted for detecting the occurrence of fault that occurred anywhere in the grid. Classification of the type of fault occurred is achieved from the spectral coefficients with the aid of artificial intelligence. The proposed system can considerably assist system protection center (SPC) in fault localization and to restore the line at the earliest. Effectiveness of proposed system has been validated using case studies conducted on standard power system networks.
\end{abstract}

Keywords: Phasor measurement unit (PMU), Backup protection, Fault classification, Support vector machine (SVM), Equivalent power factor angle (EPFA)

\section{Introduction}

Real time backup monitoring system for transmission lines is quintessential for stable and reliable operation of any power grid. Such backup system plays a crucial role during power grid fault conditions and protection system breakdowns [1-5]. Hence, such methodologies are gaining much research attraction in recent years [6-11]. Emerging phasor measurement units (PMUs) facilitate realization of remote monitoring system for transmission lines using global positioning system (GPS) and wide area communication systems. Backup monitoring systems based on PMUs are being developed and tested across the globe. Major functionalities of such remote backup monitoring system are detecting and classifying the transmission line faults occurred in a power grid [12-15].

\footnotetext{
*Correspondence: jayabharat_res@yahoo.co.in

${ }^{2}$ Department of Electrical and Electronics Engineering, National Institute of Technology, Tiruchirappalli, Tamilnadu 620015, India

Full list of author information is available at the end of the article
}

Numerous researches have been presented in literature for transmission line fault detection and classification using PMUs. Major research works are enumerated here. Fault classification scheme based on SVMs for preventing incorrect operation of conventional distance relays was presented in reference [15]. However, the paper does not discuss about discrimination of faulty phases. Reference [16] proposed protection method based on PMU measurements for transposed and un-transposed transmission lines. Although the proposed scheme detects the fault, no method was presented to classify the type of fault. Fault detection, classification and location using PMU measurements was presented in reference [17]. But influence of fault resistance (FR) and fault inception angle (FIA) on fault classification was not studied. Fault location technique for multi-terminal transmission lines was presented in reference [18]. The paper did not discuss about fault classification. Technical issues associated with fault diagnosis based 
on the data acquired from intelligent electronic devices (IEDs) were discussed in reference [19]. It can be perceived from the literature that majority of the fault detection and classification methods presented are focused on specific transmission line configurations. Fault monitoring of whole power grid require execution of these methods for all transmission lines. This may not be feasible in practice or incur increased overall cost when employed in as backup protection system.

This paper proposes a remote backup fault monitoring system for detecting and classifying all types of transmission lines faults occurred anywhere in a power grid. The system can achieve the functionalities using PMU measurements at any one of the generator buses. Frequency domain analysis of equivalent power factor angle (EPFA) is employed for detecting the fault. Classification of the fault is achieved with the aid of artificial intelligence. Proposed methodologies studied and validated on WSCC-9 (Western system coordinating council) bus system and IEEE-39 bus system.

Precise detection and classification of fault occurred at any locality of a power grid with minimal measurements form the major contribution of this paper. This is a distinctive advantage compared to many of the existing methods which have limited transmission line configurations as mentioned before. The minimal requirements on measurements make the proposed method ideal for providing backup monitoring. Moreover, the proposed methodology is insensitive to FIA and has minor sensitivity to FR. The proposed methodology can significantly contribute SPC for accelerated fault localization and restoration of faulty line.

\section{Introduction to EPFA and its dependence on transmission line faults}

Concept of EPFA and its variations during transmission line faults are discussed in this section. Angular difference between equivalent three-phase voltage phasor (Vs) and equivalent three-phase current phasor (Is) is termed as EPFA (Фeq). The concept of EPFA and its measurement are illustrated below with the help of Fig. 1.

From Fig. 1, the axes $d_{s}$ and $q_{s}$ represent the stationary reference frame in which PMU measurements of three phase voltage phasors $\left(V_{R}, V_{Y}, V_{B}\right)$ and current phasors $\left(I_{R}, I_{Y}, I_{B}\right)$ are shown. The autonomous reference frame $d_{s}^{e}$ and $\mathrm{q}_{\mathrm{s}}^{\mathrm{e}}$ rotate at a constant speed corresponding to nominal system frequency $\left(\omega_{\mathrm{s}}\right)$. During normal operating conditions, both system frequency $(\omega)$ and $\omega_{\mathrm{s}}$ are equal. Mathematical

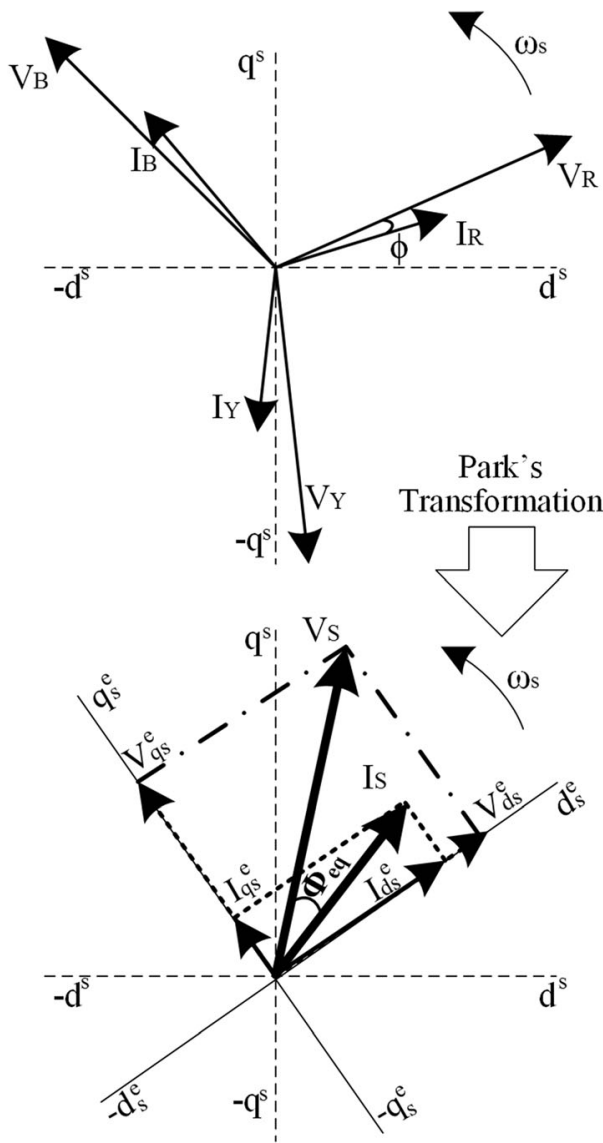

Fig. 1 Phasor diagram illustrating the power factor angle and EPFA

formulation of $d_{s}^{e}$ and $\mathrm{q}_{\mathrm{s}}^{\mathrm{e}}$ axis components of voltage and current are as presented in references [20, 21].

The angular separation between equivalent voltage phasor $\left(\overrightarrow{V_{s}}\right)$ and equivalent current phasor $\left(\overrightarrow{I_{s}}\right)$, called EPFA $\left(\Phi_{\text {eq }}\right)$, can be calculated using eq. (1).

$$
\Phi=\tan ^{-1}\left(\frac{\mathrm{V}_{\mathrm{qs}}^{\mathrm{e}}}{\mathrm{V}_{\mathrm{ds}}^{\mathrm{e}}}\right)-\tan ^{-1}\left(\frac{\mathrm{I}_{\mathrm{qs}}^{\mathrm{e}}}{\mathrm{I}_{\mathrm{ds}}^{\mathrm{e}}}\right)
$$

EPFA holds a constant value during normal operating conditions, as the system frequency and rotating frame frequencies are equal. During fault conditions in a transmission line, this relation gets violated and EPFA undergoes variation, especially when measured at generator terminals. This can be justified as follows; during transmission line fault condition, the topology of the three-phase system gets altered and the total reactance seen by the generator changes. Transient and sub-transient reactances play their respective roles within the synchronous generator followed by the contingency. Line current waveform distortion resulting from fault generates the harmonic frequencies in the current. As the fault current has to 


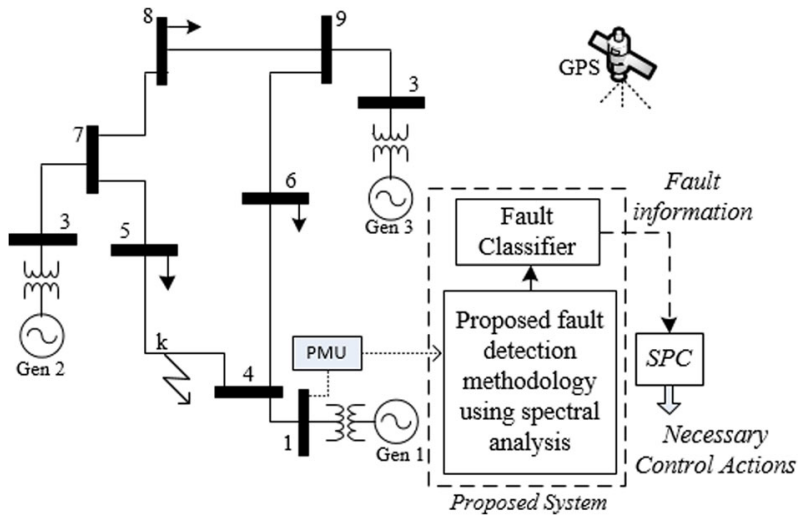

Fig. 2 Proposed fault classification methodology

a
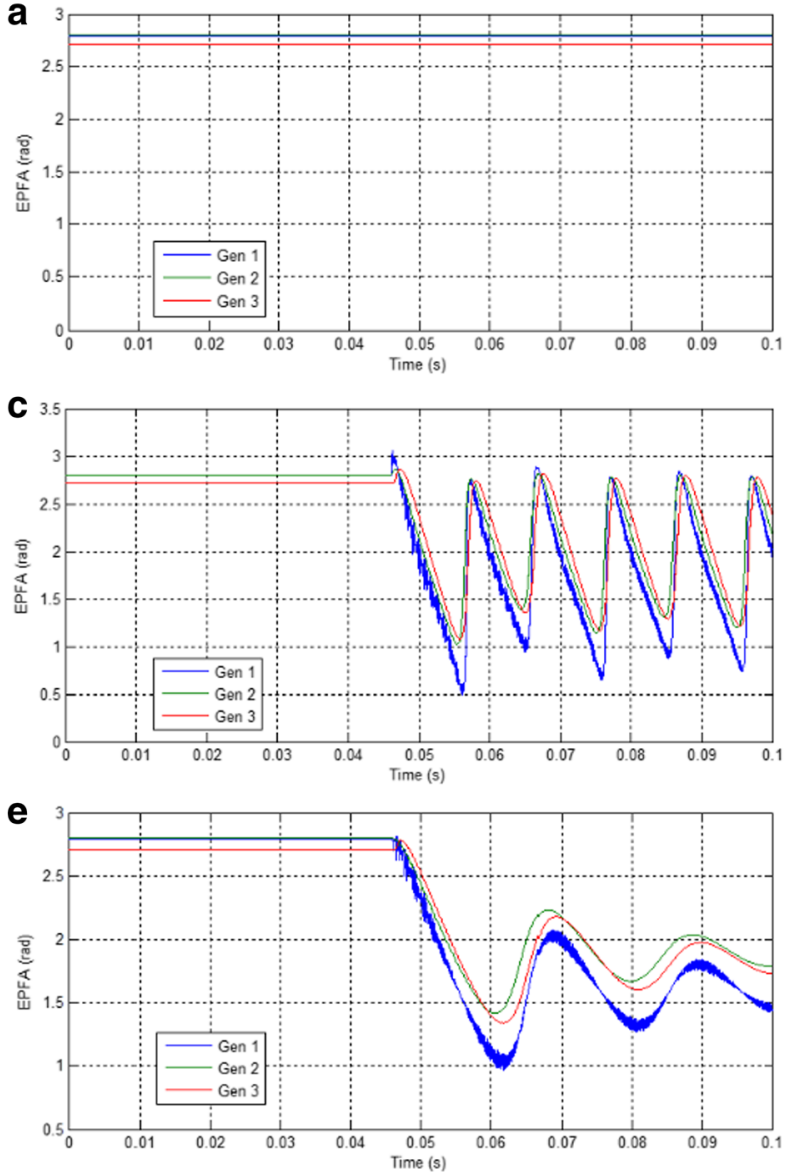

flow from generator to fault location through the transmission line, harmonic voltages get developed due to line impedances. These voltage and current harmonics along with change in overall system reactances trigger variations in the power factor angle at generator terminals $(\phi)$ and hence in EPFA $\left(\Phi_{\text {eq }}\right)$. It can be observed from the case studies (as illustrated in the following section of this paper) that frequencies related to EPFA variation have hidden fault information. These variations are analysed in frequency domain and the coefficients acquired are utilized in this paper for real time fault monitoring.

\section{Proposed fault monitoring methodology based on EPFA}

The proposed fault monitoring methodology, with which transmission line fault can be detected and classified, is elaborated in this section. The spectral coefficients of
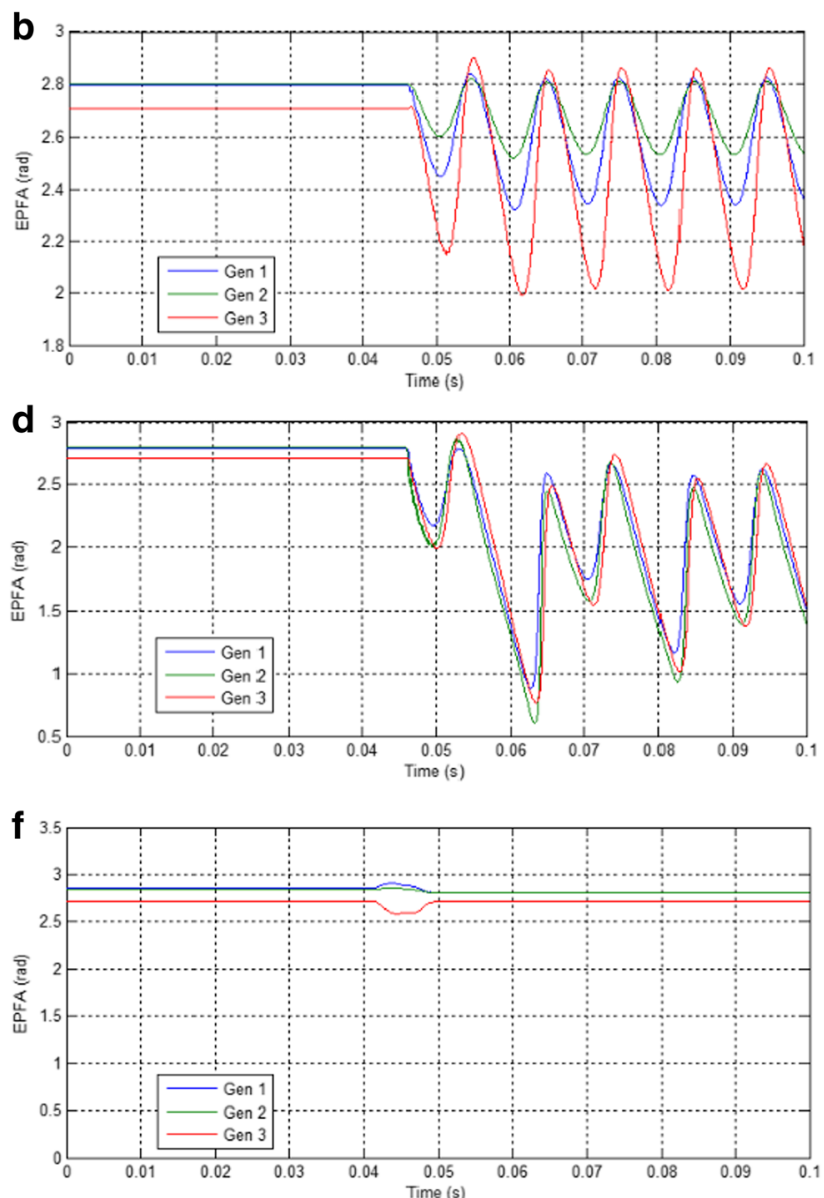

Fig. 3 a EPFA variation during no-fault condition in WSCC-9bus system. b. EPFA variation during LG-Fault condition in WSCC-9bus system with $F R=0 \Omega$ and FIA $=0^{\circ}$. c. EPFA variation during LL-Fault condition in WSCC-9bus system with FR $=$ $0 \Omega$ and FIA $=0^{\circ} .3$ d. EPFA variation during LLG-Fault condition in WSCC-9bus system with FR $=0 \Omega$ and FIA $=0^{\circ}$. e. EPFA variation during LLL-Fault condition in WSCC-9bus system with $F R=0 \Omega$ and FIA $=0^{\circ}$. f. EPFA variation during wide area load change in WSCC-9bus system 


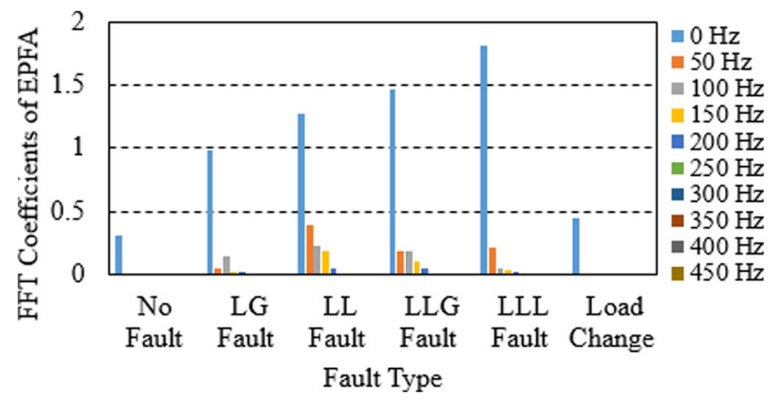

Fig. 4 FFT spectra of EPFA variation shown in Fig. 3(a) to $(f)$

EPFA $\left(\chi_{k}^{\phi}\right)$ are extracted through FFT analysis of EPFA variation as given in eq. (2).

$$
\chi_{k}^{\phi}=\left(\frac{1}{N}\right) \sum_{n=0}^{N-1} \Phi_{e q}(n) e^{\frac{-2 \pi k n}{N}} 0<k<N-1
$$

In eq. 6, $\mathrm{N}$ stands for number of samples. Online fault detection is carried out from these spectral coefficients and classification of the fault is achieved with the aid of nominal voltage coefficients (NVCs) using multi-class SVM as explained in following section. Spectral analysis of EPFA during various operating conditions are studied through case studies conducted on standard WSCC-9 bus system. Functional block diagram of system under study is shown in Fig. 2. WSCC-9 bus system with three load buses modeled as constant power has been taken for case study [15]. The transmission lines parameters are taken as presented in reference [22]. All three synchronous generators (G1 to G3) are modeled with sixth order having steam turbine governor and IEEE type-1 exciter. All generators are assumed to be having same rating. It is assumed that all computational and communication facilities are available for the proposed scheme.

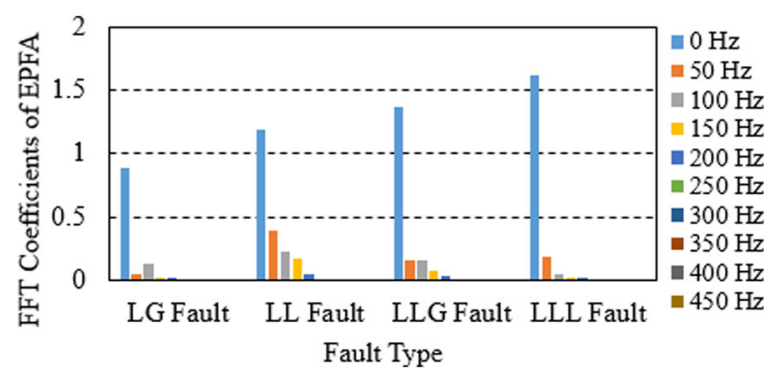

Fig. 5 FFT spectra of EPFA variation with fault conditions of $\mathrm{FR}=10 \Omega, \mathrm{FIA}=0^{\circ}$ in WSCC-9bus system

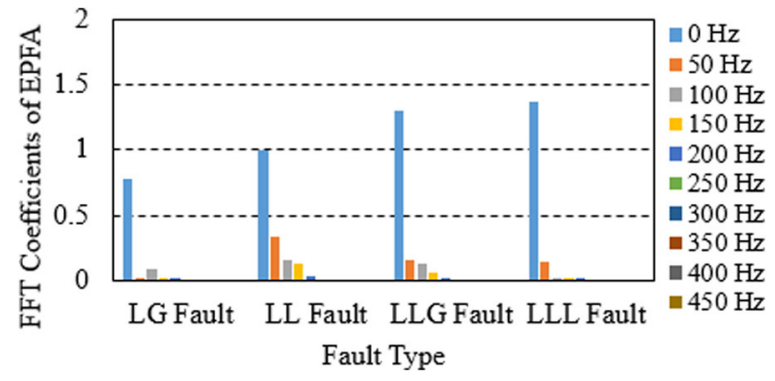

Fig. 6 FFT spectra of EPFA variation with fault conditions of $\mathrm{FR}=100 \Omega, \mathrm{FIA}=0^{\circ}$ in WSCC-9bus system

The transmission line connecting buses 4 and 5 is taken for case study in this section (this branch is equivalent to a doubly fed transmission line). Faults were created one at a time at $20 \mathrm{~km}$ from Bus 4. The EPFA of generator Gen-1 is opted for fault monitoring. During normal operating conditions, the EPFA of Gen-1 is shown in Fig. 3(a), and has a constant value. While LG fault happened in branch 4-5 (20 km away from bus 4) at $0.04 \mathrm{~s}$ with FR of zero ohms and FIA of zero degrees, the EPFA variation is as shown in Fig. 3(b). It can be observed that till fault time, EPFA was constant and after occurrence of fault it started varying. EPFA variation during LL, LLG and LLL faults are shown in Fig. 3(c) to (e) respectively. Variations in EPFA during load change is also studied and plotted in Fig. 3(f) (EPFA of all buses are shown for illustrating the impact of fault on all buses. Proposed methodology necessitate EPFA variation at any one of the generator buses).

The spectral analysis of these EPFA variations are conducted using FFT and the coefficients estimated are shown in Fig. 4.

To verify the impact of FR and FIA on EPFA variation, the same test conditions (Fault created between buses 4 and 5 at $20 \mathrm{~km}$ from bus 4) is taken with FR of $10 \Omega, 100 \Omega$ and FIA of $0^{\circ}, 45^{\circ}, 120^{\circ}$ are studied. FFT spectra for these test conditions are shown in Figs. 5, 6, 7 and 8 respectively.

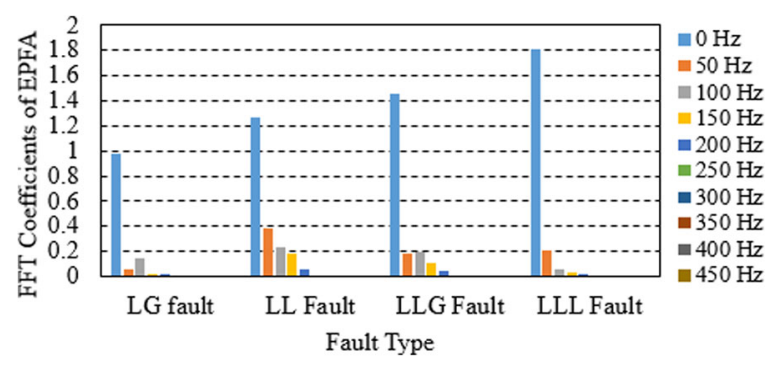

Fig. 7 FFT spectra of EPFA variation with fault conditions of $\mathrm{FR}=0 \Omega, \mathrm{FIA}=45^{\circ}$ in WSCC-9bus system 


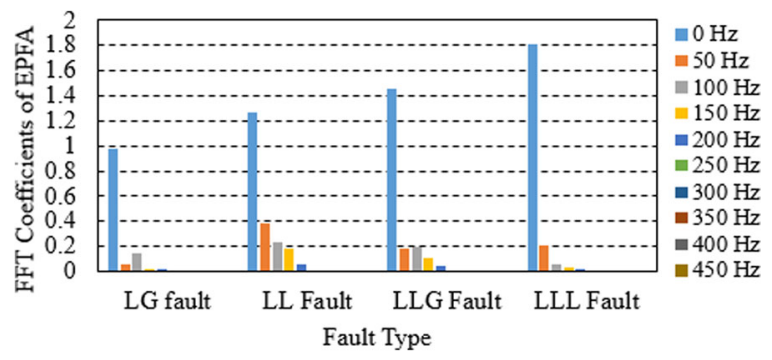

Fig. 8 FFT spectra of EPFA variation with fault conditions of $\mathrm{FR}=0 \Omega, \mathrm{FIA}=120^{\circ}$ in WSCC-9bus system

Figures 4, 5, 6, 7 and 8 validate that, transmission line faults of any kind can result in variation of EPFA and hence reflect it as non-zero coefficients for all frequencies above zero Hertz. For example, in LG fault condition with FR of $0 \Omega$ and FIA of $0^{\circ}$ (Fig. 4), the frequency coefficients corresponding to $0 \mathrm{~Hz}$, $50 \mathrm{~Hz}, 100 \mathrm{~Hz}, 150 \mathrm{~Hz}$ and $200 \mathrm{~Hz}$ are found to be $0.98,0.05,0.14,0.01$ and 0.001 respectively. When the same fault condition occurred with FR of $10 \Omega$ the FFT spectra of EPFA variation is shown in Fig. 5. It is clear that the coefficients of frequencies $0 \mathrm{~Hz}$, $50 \mathrm{~Hz}$ and $100 \mathrm{~Hz}$ have undergone slight reduction to $0.92,0.04$ and 0.13 respectively. All other coefficients have same value. When FR is further increased to $100 \Omega$, FFT spectra of EPFA variation is shown in Fig. 6. It is evident from the figure that the frequency coefficients $0 \mathrm{~Hz}$ and $100 \mathrm{~Hz}$ are found to have undergone minor reduction to 0.87 and 0.11 respectively. When the faults are studied with FIAs of $60^{\circ}$ and $120^{\circ}$, no changes in FFT spectra of $0^{\circ}$ are observed. Since frequencies of $0 \mathrm{~Hz}, 50 \mathrm{~Hz}, 100 \mathrm{~Hz}$, $150 \mathrm{~Hz}$ and $200 \mathrm{~Hz}$ (indicated as $\sigma, \alpha, \beta, \gamma$ and $\delta$ respectively) are found to be dominant in all fault conditions, they are opted for detection and classification of fault occurred. The functional block diagram of the proposed backup fault monitoring methodology is illustrated in Fig. 9.

From Fig. 9, PMU measurements from generator bus are transferred to fault classifier through proper communication channels. Inside fault classifier module, the acquired phasors are transformed to synchronous reference frame using Park's transformation and EPFA is estimated, as illustrated in preceding section. EPFA over time is recorded in memory and FFT analysis is carried out. The estimated FFT coefficients $\sigma, \alpha, \beta, \gamma$ and $\delta$ are fed to fault detection algorithm. Fault detection algorithm detects the presence of fault based on the values of $\alpha$ and $\beta$. Values of these coefficients above zero is the clear indication of fault occurred on a transmission line.

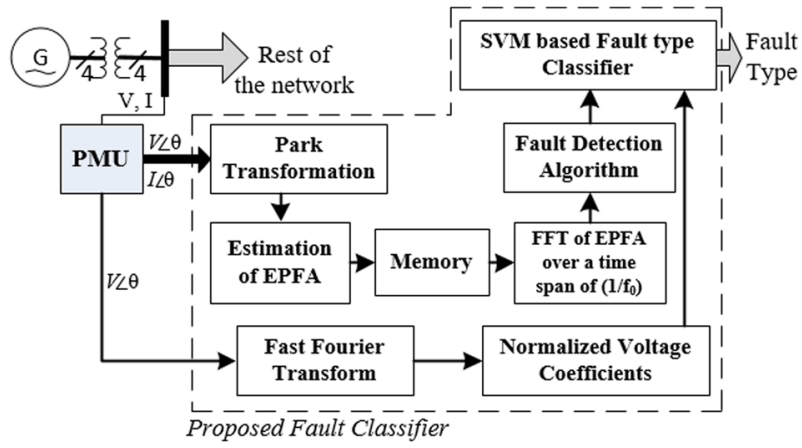

Fig. 9 Schematic diagram of fault classifier module in the proposed backup fault monitoring system

After detecting the fault, the FFT coefficients $\sigma, \alpha, \beta$, $\gamma$ and $\delta$ are transferred to SVM classifier module. The module can classify type of fault from these coefficients, however, the phase discrimination cannot be achieved. This is due to the loss of phase information related to fault when converting the threephase phasors to synchronous reference frame. Hence SVM classifier necessitates some auxiliary parameters for phase discrimination that have the information regarding the faulty phase, like voltage coefficients, current coefficients or power coefficients. This paper utilizes NVCs ( ГR, ГY, ГВ) for phase discrimination as given in eq. (3).

$$
\Gamma_{\mathrm{A} / \mathrm{B} / \mathrm{C}}=\frac{\eta_{\mathrm{A} / \mathrm{B} / \mathrm{C}}^{50}}{\max \left\{\eta_{A}^{50}, \eta_{B}^{50}, \eta_{C}^{50}\right\}}
$$

Where, $\eta_{i}^{50}$ denotes the FFT coefficient of $50 \mathrm{~Hz}$ frequency component in i-phase voltage. The choice of $\eta_{i}^{50}$ for estimating NVCs can be validated based on the case studies conducted on the test system shown in Fig. 2. NVCs estimated using $\eta_{i}^{0}, \eta_{i}^{50}, \eta_{i}^{100}$ and $\eta_{i}^{150}$ for no-fault condition and four fault conditions in WSCC system shown in Fig. 2 are tabulated in Table 1. A balanced harmonic-free system is considered for the study. During no-fault condition NVCs estimated using $\eta_{i}^{50}$ will have unity values indicating equal values of fundamental voltages in all phases. NVCs estimated using $\eta_{i}^{0}, \eta_{i}^{100}$ and $\eta_{i}^{150}$ have zero values, since the system is assumed to be free of harmonics. All faults are applied with FR of $0 \Omega$ and FIA of $0^{\circ}$, one at a time at $80 \mathrm{Km}$ away from Gen-1 in branch 4-5 of WSCC system shown in Fig. 2. During fault condition, NVCs estimated using $\eta_{i}^{50}$ differ in their values based on the type of fault and NVCs estimated using all others remain zero.

Hence SVM classifier uses two inputs viz. frequency coefficients of EPFA variation $(\sigma, \alpha, \beta, \gamma$ and $\delta$ ) and 
Table 1 NVCs for phase discrimination in the test system shown in Fig. 1

\begin{tabular}{llllll}
\hline Type of FFT coefficient & $\begin{array}{l}\text { No Fault } \\
\left(\Gamma_{\mathrm{R},}, \Gamma_{\mathrm{Y}}, \Gamma_{\mathrm{B}}\right)\end{array}$ & $\begin{array}{l}\text { LG Fault (AG) } \\
\left(\Gamma_{\mathrm{R},}, \Gamma_{\mathrm{Y}}, \Gamma_{\mathrm{B}}\right)\end{array}$ & $\begin{array}{l}\text { LL Fault (AB) } \\
\left(\Gamma_{\mathrm{R},}, \Gamma_{\mathrm{Y},}, \Gamma_{\mathrm{B}}\right)\end{array}$ & $\begin{array}{l}\text { LLG Fault (ABG) } \\
\left(\Gamma_{\mathrm{R},}, \Gamma_{\mathrm{Y}}, \Gamma_{\mathrm{B}}\right)\end{array}$ & $\begin{array}{l}\text { LLL Fault (ABC) } \\
\left(\Gamma_{\mathrm{R},}, \Gamma_{\mathrm{Y}}, \Gamma_{\mathrm{B}}\right)\end{array}$ \\
\hline$\eta_{i}^{0}$ & $0,0,0$ & $0,0,0$ & $0,0,0$ & $0,0,0$ & $0,0,0$ \\
$\eta_{i}^{50}$ & $1,1,1$ & $0.6,1,1$ & $0.6,0.6,1$ & $0.5,0.5,1$ & $1,1,1$ \\
$\eta_{i}^{100}$ & $0,0,0$ & $0,0,0$ & $0,0,0$ & $0,0,0$ & $0,0,0$ \\
$\eta_{i}^{150}$ & $0,0,0$ & $0,0,0$ & $0,0,0$ & $0,0,0$ & $0,0,0$ \\
\hline
\end{tabular}

NVCs estimated using $\eta_{i}^{50}$. Details of the SVM classifier is described in the following subsection.

Since the FFT coefficients and NVCs are interspersed, a simple logical function may not able to accomplish the classification of fault. Hence artificial intelligence technique with fine learning capability to be adopted. SVM classifiers are becoming a predominant machine intelligence technique for wide range of applications in power systems [23-25]. SVM classifiers are well defined in the literature [23, 24, 26]. A brief outline of SVM classifiers from the perspective of fault classification in SPG is illustrated below.

SVM classifier can be either one-class SVM or multi-class SVM. The former can classify a single type of data from a bunch of data, while the latter can classify multiple types of data. Both approaches make a linear decision surface that secede data classes with maximum distance from border line (support vectors). When such linear decision surface cannot be achieved, then SVM draws the data to a higher dimensional feature space using kernel function so that a separating linear decision surface exists can be determined [23, 24, 26].

SVM finds the optimal separating hyperplane from the training data set near to the border line, helps to generalize the data for unseen cases $[23,24,26]$. LIBSVM toolbox in MATLAB/SIMULINK environment with radial basis function (RBF) kernel is deployed in this research work. The kernel function is defined as follows;

$$
K\left(x_{i}, x_{j}\right)=\exp \left(-\psi\left\|x_{i}-x_{j}\right\|\right)^{2}
$$

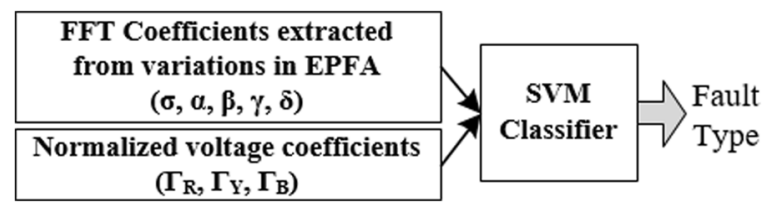

Fig. 10 Functional block diagram of SVM classifier for fault classification
Where, $\psi$ is the kernel parameter that defines nonlinear mapping and $\mathrm{x}_{\mathrm{i}}$ stands for $\mathrm{i}^{\text {th }}$ input vector.

Frequency coefficients of EPFA variations $(\sigma, \alpha, \beta, \gamma$ and $\delta)$ and NVCs (ГR, ГY, ГВ) form the input data set. Fault type data corresponding to each set of values in input data set constitutes the output data set for SVM classifier. SVM classifier is trained with 1800 data sets to afford better boundary for fault classification. The input data sets are generated for different fault locations, fault resistances and inception angles. Since fault types are of many classes, one-step multi-class SVMs are employed for fault classification [23, 24, 26]. Schematic diagram of SVM classifier for fault classification is shown in Fig. 10. SVM algorithm used is as presented in references [23, 24, 26].

The diversity in training data has been maintained by keeping the data for distinct fault locations having different FRs and FIAs.

\section{Results and discussion}

Simulation studies and the results observed in proposed backup fault monitoring scheme are discussed in this section. IEEE-39 bus system $(400 \mathrm{kV})$ is opted for case study, as shown in Fig. 11 [15]. All ten conventional generators (G1 to G10) generators and transmission lines are modelled in the similar way as in case-I. EPFA of all generators during normal operating conditions are shown in Fig. 12(a). The constant value of EPFA during

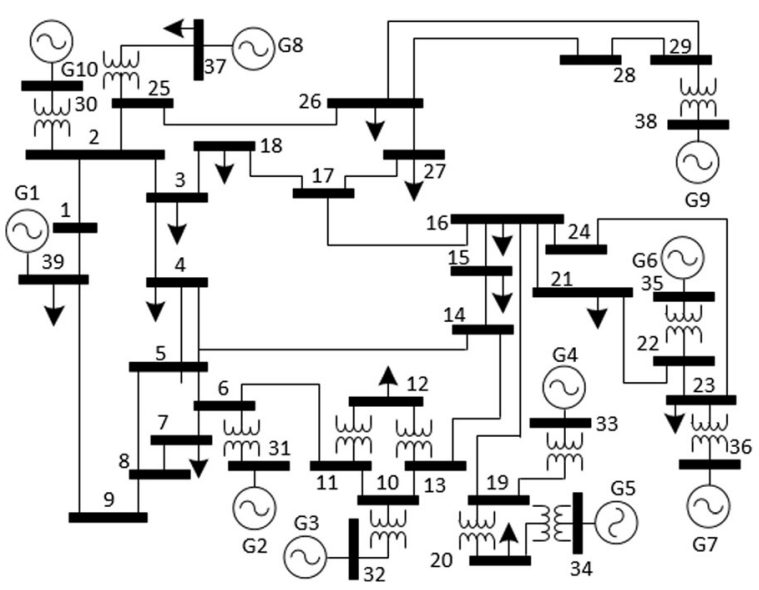

Fig. 11 Single line diagram of IEEE-39 bus system 

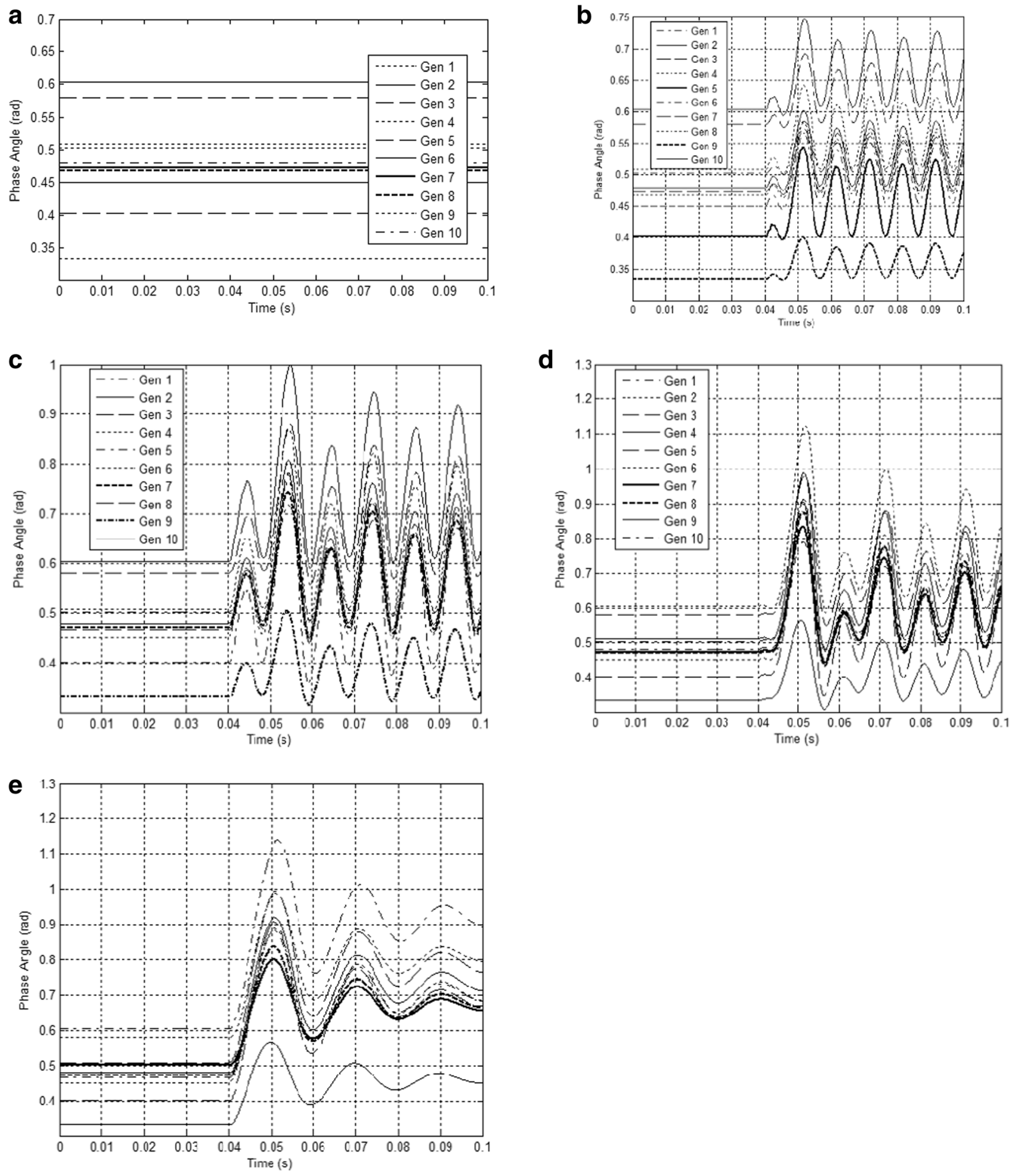

Fig. 12 a. EPFA variation during no-fault condition in IEEE-39 bus system. b. EPFA variation during LG-fault (AG) condition at branch 3-18, $50 \mathrm{~km}$ away from bus-3 with $\mathrm{FR}=0 \Omega$ and $\mathrm{FIA}=0^{\circ}$ in IEEE-39 bus system. c. EPFA variation during LL-fault (BC) condition at branch 4-5 $20 \mathrm{~km}$ away from bus-4, with $F R=10 \Omega$ and $F I A=45^{\circ}$ in IEEE-39 bus system. $\mathbf{d}$. EPFA variation during LLG-fault (CAG) condition at branch 23-24, $70 \mathrm{~km}$ away from bus-23 with FR $=100 \Omega$ and FIA $=120^{\circ}$ in IEEE-39 bus system. 12 e. EPFA variation during LLL-fault (ABC) condition at branch 5-6, $50 \mathrm{~km}$ away from bus-5 with $\mathrm{FR}=0 \Omega$ and FIA $=0^{\circ}$ in IEEE-39 bus system 


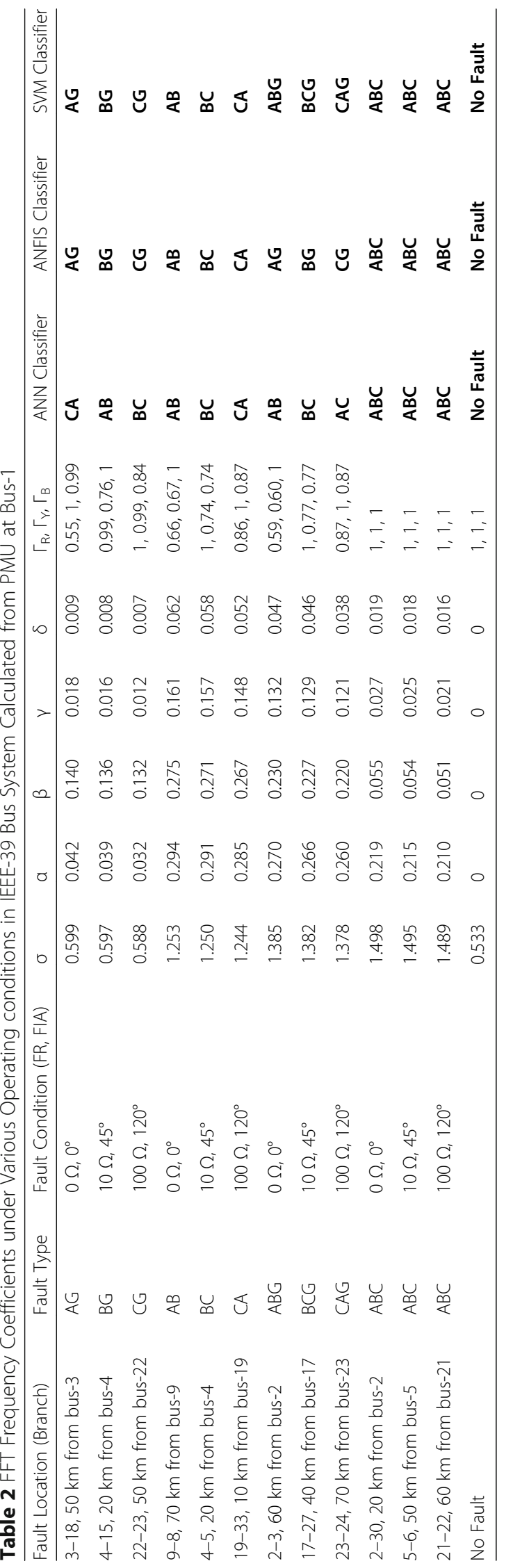


this condition is reflected as value of 0.533 for $\sigma$ and a value of zero for all other frequency coefficients as given in Table 2. EPFA variations corresponding to various fault conditions applied at random locations of the system are shown in Fig. 12(b)-(e) (EPFA of all generators are shown in figure for validating the impact of fault on all generators. Proposed methodology require EPFA of any one of the generators alone). Frequency coefficients of EPFA variations in fault conditions are listed in Table 2. For example, EPFA variations of all generators while LG-fault (AG) on transmission line connecting buses 3 and 18 at $50 \mathrm{~km}$ away from bus-3 with FR of $0 \Omega$ and FIA of $0^{\circ}$ is shown in Fig. 12(b). Time of occurrence of fault is $0.04 \mathrm{~s}$, and hence, up to $0.04 \mathrm{~s}$ EPFAs of all generators have constant value and starts varying afterwards. The frequency coefficients $\sigma, \alpha, \beta, \gamma$ and $\delta$ have the values of $0.599,0$. 042, 0.140, 0.018, 0.009 as given in Table 2. Similarly, EPFA variation during LL, LLG and LLL are shown in Fig. 12(c)-(e) and corresponding frequency spectral coefficients are listed in Table 2. It is evident from the Figs. 12(a)-(e) and Table 2 that EPFA suffers variation during faults and the corresponding frequency components depend on type of fault occurred. It can be observed that, for fault occurred far away point from the generator causes frequency coefficients in EPFA variation. However, the magnitudes of the EPFA frequency components vary with fault location and this demands the necessity for artificial intelligence to classify the fault type. The frequency components and corresponding NVCs (as given in Table 2) are given to SVM module and the results of the fault classification are also given in Table 2. As a comparative study with others classifiers, result obtained with ANFIS and ANN classifiers are described in the succeeding subsection.

\subsection{Comparative study with other machine intelligence techniques}

Study on performance accuracy of SVM classifier compared to artificial neural network (ANN) and adaptive neuro-fuzzy inference system (ANFIS) are illustrated in this section. The same test case of IEEE-39 bus system is considered for comparative study. ANN has been developed with feed-forward back propagation method consisting of two layers of input and output with two hidden intermediate layers [27]. While ANFIS based fault classifier was developed using hybrid optimization method with 600 membership functions [28]. The inputoutput data set is made common for ANN, ANFIS and SVM classifiers. The results after case studies are tabulated in Table 2, which validate the superiority of SVM classifiers over the others.
From Table 2, ANN can precisely classify LL faults and LLL faults at random locations of the system. However, it has misclassified all LG and LLG faults. While, ANFIS classifier classified LG, LL and LLL faults but classification result went wrong on LLG faults. SVM has classified all fault conditions and hence it can be presumed that SVM classifier is superior to other two for proposed fault classification methodology.

\section{Conclusion}

Backup protection of transmission lines is of elevated significance in power grid operation and control. Although advanced numerical relays are available for transmission line protection, backup protection is important to provide adequate system performance during protection system breakdowns and circuit breaker failures. This paper proposes a fault monitoring methodology can be adopted as backup monitoring in power grids. The proposed system can detect and classify transmission line fault occurred anywhere in the power grid with the aid of PMUs. Since any one of the generator bus PMU measurements are sufficient, the methodology is ideal for providing backup protection. This is unique advantage compared to conventional methods which are devised for specific transmission line configurations like transmission line fed at single end or double ends.

\section{Authors' contributions}

GP has developed and implemented the proposed algorithm. MB has made substantial contributions to simulate WSCC-9 bus system. MJBR has been the technical adviser for the total work and DKM has supported us in interpreting the simulation results for fault analysis using PMU measurements. All authors read and approved the final manuscript.

\section{Competing interests}

The authors declare that they have no competing interests.

\section{Author details}

${ }^{1}$ Department of Electrical Engineering, National Institute of Technology, Calicut, Kerala, India. ${ }^{2}$ Department of Electrical and Electronics Engineering, National Institute of Technology, Tiruchirappalli, Tamilnadu 620015, India. ${ }^{3}$ Department of Electrical and Electronics Engineering, BITS Mesra, Ranchi, India.

Received: 4 September 2017 Accepted: 8 May 2018

Published online: 05 June 2018

\section{References}

1. Mallikarjuna, B., Vardhan Varma, P. V., Samir, S. D., Jaya Bharata Reddy, M., \& Mohanta, D. K. (2017). An adaptive supervised wide-area backup protection scheme for transmission lines protection. Protection and Control of Modern Power Systems, 22(2), 1-16.

2. Wang, J., Zhong, H., Xia, Q., et al. (2015). Transmission network expansion planning with embedded constraints of short circuit currents and $\mathrm{N}-1$ security. Journal of Modern Power Systems and Clean Energy, 3(3), 312-320.

3. Jamil, M., Sharma, S. K., \& Singh, R. (2015). Fault detection and classification in electrical power transmission system using artificial neural network. SpringerPlus, 334(4), 1-13.

4. Majd, A. A., Samet, H., \& Ghanbari, T. (2017). k-NN based fault detection and classification methods for power transmission systems. Protection and Control of Modern Power Systems, 2, 32, 1-11. 
5. Roostaee, S., Mini, S. T., \& Mehfuz, S. (2017). Experimental studies on impedance based fault location for long transmission lines. Protection and Control of Modern Power Systems, 2:16, 1-9.

6. Slootweg, J. G., \& Kling, W. L. (2002). Impacts of distributed generation on power system transient stability. Proc IEEE Power Eng Soc Summer Meet, 2, 862-867.

7. Cecati, C., Citro, C., \& Siano, P. (2011). Combined operations of renewable energy systems and responsive demand in a smart grid. IEEE Transactions on Sustainable Energy, 2(4), 468-476.

8. Gopakumar, P., Jaya bharata Reddy, M., \& Mohanta, D. K. (2014). Letter to the editor: Stability concerns in smart grid with emerging renewable energy technologies. Electric Power Components and Systems, 42(3), 418-425.

9. Ma, J., Zhang, P., Fu, H., Bo, B., Dong, Z. (2010). Application of phasor measurement unit on locating disturbance source for low-frequency oscillation. IEEE Transactions on Smart Grid, 3(1), 340-346.

10. Seethalekshmi, K., Singh, S. N., \& Srivastava, S. C. (2011). A Synchrophasor assisted frequency and voltage stability based load shedding scheme for self-healing of power system. IEEE Transactions on Smart Grid, 2(2), 221-230.

11. Hashiesh, F., Mostafa, H. E., Khatib, A., Helal, I., \& Mansour, M. M. (2012). An intelligent wide area Synchrophasor based system for predicting and mitigating transient instabilities. IEEE Transactions on Smart Grid, 3(2), 645-652.

12. Phadke, A. G., Thorp, J. S., \& Adamiak, M. G. (1983). A new measurement technique for tracking voltage phasors, local system frequency, and rate of change of frequency. IEEE Transactions on Power Apparatus and Systems, 102(5), 1025-1038

13. Phadke, G., \& Thorp, J. S. (2008). Synchronized Phasor Measurements and Their Applications. Power electronics and power systems series. New York: Springer Publication.

14. NERC Technical Reference Document (2010) Transmission system backup protection systems, Dec 2010

15. Seethalekshmi, K., Singh, S. N., \& Srivastava, S. C. (2012). A classification approach using support vector machines to prevent distance relay Maloperation under power swing and voltage instability. IEEE Transactions on Power Delivery, 27(3), 1124-1133.

16. Shan, C., Chih-Wen, L., \& Joe-Air, J. (2002). A new adaptive PMU based protection scheme for transposed/Untransposed parallel transmission lines. IEEE Transactions Power Delivery, 17(2), 902-907.

17. Joe-Air, J., Ching-Shan, C., \& Chih-Wen, L. (2003). A new protection scheme for fault detection, direction discrimination, classification, and location in transmission lines. IEEE Transactions Power Delivery, 18(1), 34-42.

18. Chih-Wen, L., Kai-Ping, L., Ching-Shan, C., \& Joe-Air, J. (2008). A universal fault location technique for $\mathrm{N}$-terminal $(\mathrm{N} \geq 3)$ transmission lines. IEEE Transactions Power Delivery, 23(3), 1366-1373.

19. Kezunovic, M. (2011). Smart fault location for smart grids. IEEE Transactions on Smart Grid, 2(1), 11-22.

20. Grainger, J. J., \& Stevenson, W. D. (2003). Power system analysis. New Delhi Tata McGraw-Hill Edition

21. Kundur, P. (2012). Power system stability and control. New Delhi: Tata McGraw Hill Education Pvt Ltd.

22. Gopakumar, P., Jaya Bharata Reddy, M., \& Mohanta, D. K. (2015). Transmission line fault detection and localisation methodology using phasor measurement unit measurements. IET Generation Transmission and Distribution, 11(9), 1033-1042.

23. Ravikumar, B., Thukaram, D., \& Khincha, H. P. (2010). Comparison of multiclass SVM classification methods to use in a supportive system for distance relay coordination. IEEE Transactions on Power Delivery, 25(3), 1296-1305.

24. Kalyani, S., \& Shanti Swarup, K. (2011). Classification and assessment of power system security using multiclass SVM. IEEE transactions on Systems, Man, and Cybernetics-Part C: Applications and Reviews, 41(5), 753-758.

25. Chang, C. C., \& Lin, C. J. (2011). LIBSVM: a library for support vector machines. ACM Transactions on Intelligent Systems and Technology, 2, 27:1-27:27. Software available at http://www.csie.ntu.edu.tw/ cjlin/libsvm.

26. Vapnik, V. (1998). Statistical learning theory. New York: Wiley.

27. Seyedtabaii, S. (2012). Improvement in the performance of neural networkbased power transmission line fault classifiers. IET Generation Transmission and Distribution, 6(3), 731-737.

28. Jaya Bharata Reddy, M., \& Mohanta, D. K. (2008). Adaptive-neuro-fuzzy inference system approach for transmission line fault classification and location incorporating effects of power swings. IET Generation Transmission and Distribution, 2(2), 235-244.

\section{Submit your manuscript to a SpringerOpen ${ }^{\circ}$ journal and benefit from:}

- Convenient online submission

- Rigorous peer review

- Open access: articles freely available online

- High visibility within the field

- Retaining the copyright to your article

Submit your next manuscript at $\boldsymbol{\nabla}$ springeropen.com 